\title{
Reappraisal of ultrasound imaging of neonatal intravascular catheters
}

\author{
R J Madar, S A Deshpande
}

\begin{abstract}
Ultrasonography was successful in localising the tips of neonatal intravascular catheters and showed their precise relation to the cardiac chambers and major aortic branch vessels in 28 infants. Wherever adequate resources and training are available, this technique may provide a cheaper and non-invasive option, as well as giving additional information, than can be obtained from radiography.

(Arch Dis Child 1996;75:F62-F64)
\end{abstract}

Keywords: ultrasonography, percutaneous intravascular catheters, umbilical catheters.

The use of intravascular catheters has facilitated invasive monitoring and parenteral nutritional support in critically ill neonates. Optimal positioning of these catheters is important for accurate monitoring of parameters such as central venous pressure, and for prevention of complications such as renal arterial thrombosis. Traditionally, radiography is used to check the position of the catheters, relying on certain vertebral landmarks to indicate a presumed intravascular location of the catheter tip, ${ }^{12}$ although the use of sonography for positioning of umbilical arterial and venous catheters has been suggested. ${ }^{34}$

The infants in whom these lines are placed are often premature and critically ill, requiring intensive care support. Taking $x$-rays subjects them to handling and exposure, as well as requiring the use of ionising radiation. In theory, ultrasonography has advantages over radiography, because it is non-invasive and requires minimal manipulation of the infant.
We therefore assessed the ability of crosssectional ultrasonography to visualise the intravascular catheters and locate the catheter tips.

\section{Methods}

Twenty eight infants (median (range) birthweight 1210 (640-3530) g; median (range) gestation 28 (23-40) weeks) admitted to the special care baby unit at the Royal Victoria Infirmary, Newcastle upon Tyne, and who required intravascular catheters, were enrolled in the study between June 1994 and January 1995. After the placement of an intravascular catheter its position was checked by a plain radiograph of chest, abdomen, or both. An ultrasound assessment of the catheter was performed by a single operator who was unaware of the radiographic assessment of the line position. Ultrasound studies were performed using a Hewlett Packard Sonos 100 (Hewlett Packard, UK) with a 7.5 MHZ mechanical sector scanner, and all examinations were recorded on a videotape. If line positions were subsequently adjusted, repeat $x$-ray and ultrasound examinations were carried out. The position of the line tip was assessed independently in relation to the vertebral landmarks, and compared with contemporaneous sonographic records. Where ambiguity arose, videotape recordings were also retrospectively reviewed.

\section{Results}

A total of 40 intravascular catheters (umbilical arterial catheters $n=18$; umbilical venous catheters $n=3$, and percutaneous silastic long lines $n=19)$ were inserted in these infants. In all but two instances ultrasound examination was suc-

Accepted 10 April 1996

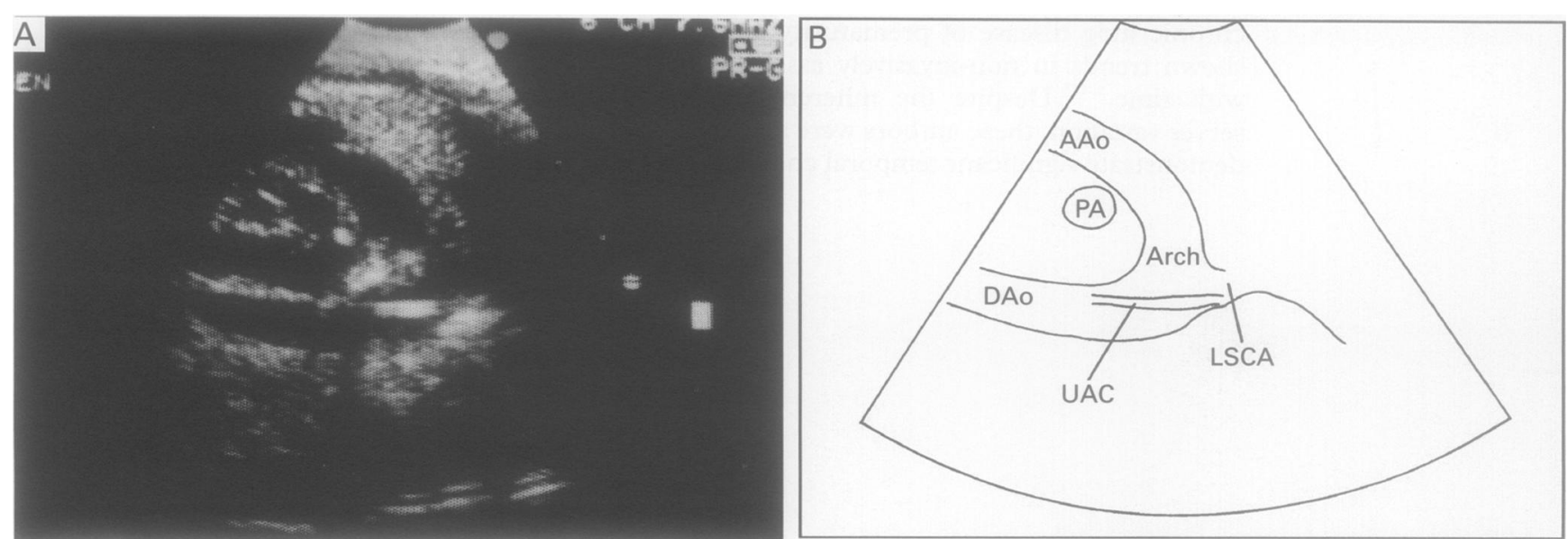

Figure 1 Suprasternal aortic arch view showing arterial catheter high in the arch towards the artery. UAC: umbilical arterial catheter; AAo: ascending aorta; Arch: aortic arch; DAo: descending aorta; FO: oval foramen; PA: pulmonary artery; LSCA lefi subclavian artery. 

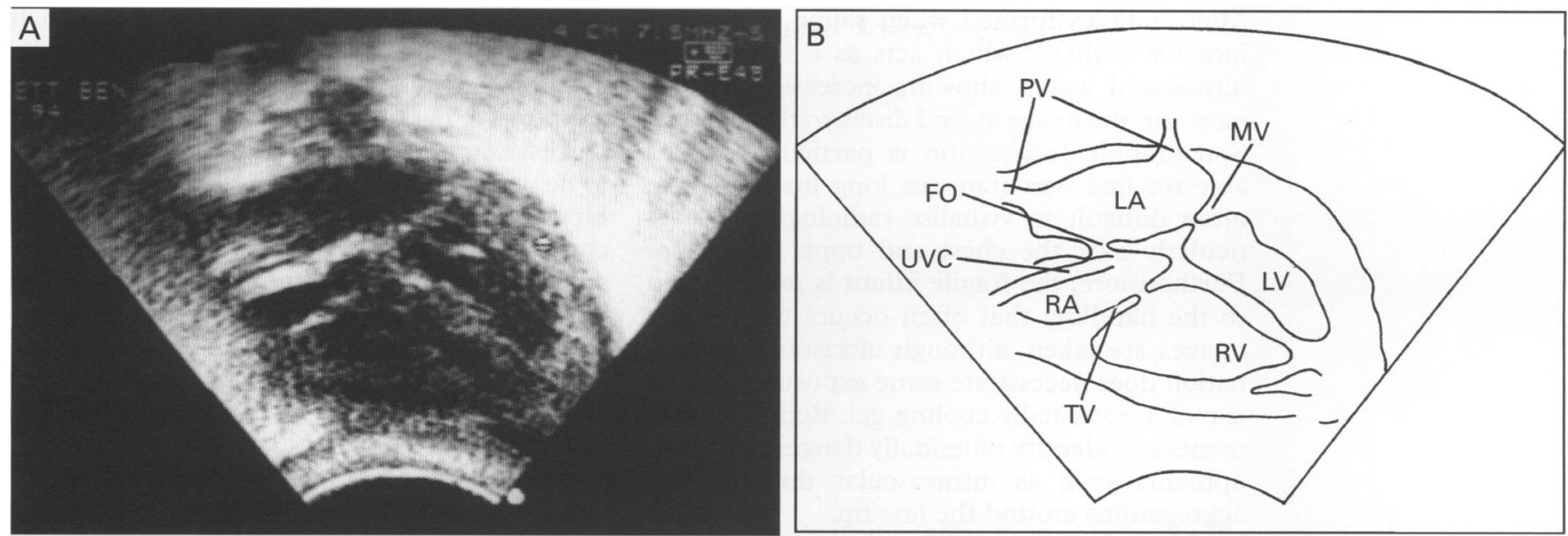

Figure 2 Subcostal four chamber view of the heart showing the tip of the umbilical venous catheter lying in the right atrium close to the tricuspid valve. UVC: umbilical venous catheter; RA: right atrium; RV: right ventricle; LA: left atrium; LV: left ventricle;TV: tricuspid valve; $M V:$ mitral valve; $P V$ : pulmonary vein; $F O$ : oval foramen.

cessful in visualising the position of the tip (fig 1), with the true intravascular position being consistent with location implied by the vertebral landmarks on $x$-rays. One infant in whom ultrasonography could not satisfactorily identify the tip of the silastic long line had a very tender abdomen, making the examination difficult; in the other infant the catheter tip was situated high in the superior vena cava. The procedure was well tolerated by all infants except the one with a tender abdomen.

Using ultrasonography, tips of venous catheters were localised to vena caval $(n=7)$, right $(n=11)$, or left atrial $(n=2)$ positions (fig 2$)$. The tip of one right atrial venous catheter lay close to the tricuspid valve and was seen to pass virtually into the right ventricle with cardiac contraction. Two venous catheters passed through the oval foramen which was not clearly evident on the $x$-ray (fig 3 ).

The precise relation of arterial catheters to the major branch vessels could be easily demonstrated by ultrasonography. In two instances the tips of the arterial catheters were seen at the origin of the left subclavian artery, while in another, the catheter just entered the ductus arteriosus. Ultrasound guidance was subsequently used in repositioning four cath- eters. In one infant aggregations were identified around the tip of the silastic long line in the right atrium; the catheter was promptly removed and the baby did not have any embolic complications.

\section{Discussion}

Ultrasonography facilities are readily available on most neonatal units where intravascular catheters such as these might be used. Our study shows that the technique can accurately locate the course of a catheter and the position of the line tip. Precise positioning of umbilical venous catheters is essential for accurate monitoring of central venous pressures and oxygen saturations. Our study shows the ease with which these lines can enter the left atrium and yet not be clearly identified on $x$-ray.

The ease of access to ultrasonography and its independence from other resources reduces exposure to ionising radiation, and the need for "out of hours" $x$-ray imaging. Use of ultrasound guidance at the time of line insertion allows the line position to be immediately adjusted, with confirmation of the amended position, obviating the need for repeated radiological evaluations. Injection of saline contrast may facilitate identification of catheter tips.
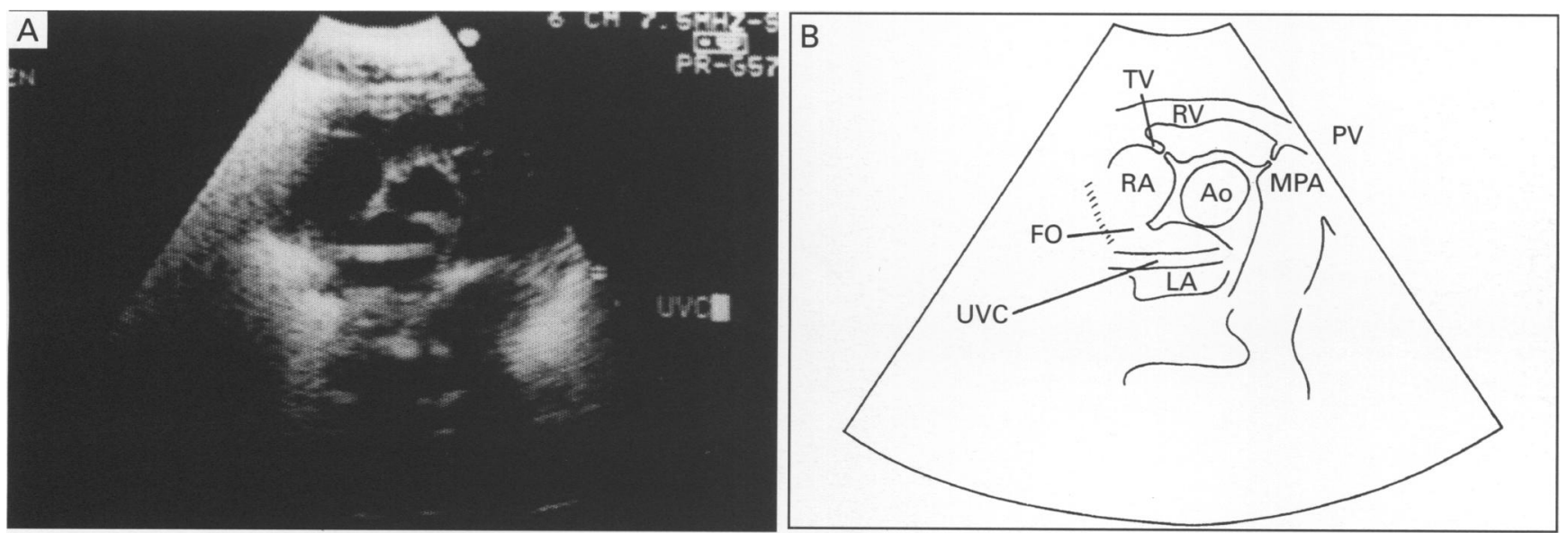

Figure 3 Parasternal short axis view of the heart, showing the umbilical venous catheter passing through the oval foramen with the tip lying in the left atrium.UVC: umbilical venous catheter; $R A$ : right atrium; RV: right ventricle; LA: left atrium; LV: left ventricle;TV: tricuspid valve;Ao: Aorta; FO: oval foramen. 
Microbubbles formed when saline is injected into the catheter which acts as a reflector to ultrasound waves, showing increased echogenicity on the image at, and distal, to the line tip. ${ }^{5}$ Sonographic localisation is particularly valuable for fine percutaneous long lines that are often difficult to visualise radiologically, particularly over the chest and upper abdomen. Furthermore, the fragile infant is not exposed to the handling that often occurs when $x$-ray images are taken, although ultrasound examination does necessitate some exposure and the use of a potentially cooling gel. Repeat assessments can identify potentially dangerous developments such as intravascular thrombi and aggregations around the line tip.

Although the suitability of ultrasonography to evaluate the position of umbilical arterial lines was reported more than a decade ago, the technique does not seem to have been widely practised. This may be due to limited definition of earlier sonographic equipment, lack of relevant expertise, and the absence of reports of ultrasound evaluation of catheters other than umbilical ones.

It is, however, necessary to have staff trained in the appropriate techniques of ultrasonography, and to ensure that the limitations of the method are realised. There are instances when ultrasonography can not reliably determine the position of the catheter and radiology is the appropriate investigation. Similarly, ultrasound localisation may be unnecessary if an $x$-ray has to be performed for another clinical indication. However, in a large proportion of cases, this cheaper and less invasive option is equally reliable and often provides more information than the traditional check $x$-ray picture.

We thank the medical illustration department of the Royal Hospital for Sick children for their help in preparing the illustrations.

1 Baker D, Berdon W, James S. Proper localisation of umbilical arterial catheters and venous catheters by lateral roentegenogram. Pediatrics 1969;43:34-9.

2 Rosen S, Reich S. Umbilical venous catheterisation in the newborn: identification of correct positioning. Radiology 1970;95:35-40.

3 Garg AK, Houston AB, Laing JM, Mackenzie JR. Positioning of umbilical arterial catheters with ultrasound. Arch ing of umbilical arterial cath

4 Greenberg M, Movahed H, Peterson B, Bejar R. Placement of umbilical venous catheters with use of bedside real-time ultrasonography. F Pediatr 1995;126:633-5.

5 Feigenbaum $H$. The echocardigraphic examination In: Feigenbaum H, ed. Echocardiography. Philadelphia: Lea \& Febiger, 1986: 50-126. 